\title{
MATHIAS FORCING WHICH DOES NOT ADD DOMINATING REALS
}

\author{
R. MICHAEL CANJAR \\ (Communicated by Thomas J. Jech)
}

\begin{abstract}
Assume that there is no dominating family of reals of cardinality $<c$. We show that there then exists an ultrafilter on the set of natural numbers such that its associated Mathias forcing does not adjoin any real which dominates all ground model reals. Such ultrafilters are necessarily $P$-points with no $Q$-points below them in the Rudin-Keisler order.
\end{abstract}

Notation and terminology. $\omega$ is the set of natural numbers. The symbols $i$, $j, k, l, m, n$ will be used exclusively to denote its elements. All our filters (and ultrafilters) are proper, nonprincipal and on $\omega$. We use the term "real" exclusively for elements of ${ }^{\omega} \omega$. We say that a real $f$ dominates a real $g$ iff $\exists m \forall n>m f(n)>g(n)$. Following [13] we let $d$ denote the minimum cardinality of a dominating family of reals and $b$ denote the minimum of an unbounded family; i.e., a family $B$ such that there is no real $g$ which dominates every real from $B . c$ is the cardinality of the continuum. With respect to forcing, we adhere to the convention that " $p \leq q$ " indicates that $p$ extends $q$. Following [1] we will write $\operatorname{Next}(X, n)$ for $\min \{x \in X \mid x \geq n\}$ when $X$ is an infinite subset of $\omega . P$ is the set of finite, increasing sequences of natural numbers:

DEFINITION 1. $P=\left\{s \in{ }^{n} \omega \mid n \in \omega \& \forall k<j<n, s(j)<s(k)\right\}$. The symbols $s$, $t$ will be used exclusively to denote elements of $P$. If $A \subseteq \omega$ and $s, t \in P$, we will write $t<(s, A)$ to indicate that $s \subseteq t$ and Range $(t-s) \subseteq A$. If $A, B$ are subsets of $\omega$, we write $(t, B) \leq(s, A)$ if $t<(s, A)$ and $B \subseteq A$. $Q_{U}$ is the Mathias notion of forcing, introduced in [10], associated with the ultrafilter $U$. It consists of the set $\{(s, A) \mid s \in P, A \in U\}$, together with the partial order $\leq$ described above. $Q_{U}$ adjoins a set $G \subseteq \omega$ which is almost a subset of every set in $U$. We will use the symbol " $G$ " exclusively to denote this set. We will write $\mathbb{\Vdash}_{U}$ to indicate forcing in $Q_{U} ; U$ will be omitted when clear from context. " $\tau$ " will be used exclusively to represent terms for reals in the $Q_{U}$-forcing language. Such terms will always be understood to be, with truth value 1 , functions in ${ }^{\omega} \omega$.

We will say that $\lambda$-c.c. partial order $Q$ is $\lambda$-nondominating if whenever $D$ is a family of reals in $V[G]$ with $|D|<\lambda$ we may find $h \in V$ so that $h$ is not dominated by any real from $D$. (In other words, $Q$ does not add small families that dominate all ground model reals.) The maximum $\lambda$ for which there exists a partial order which is $\lambda$-nondominating is, of course, $d$. A c.c.c. partial order which does not add any single real which dominates all ground models is necessarily $b$-nondominating. We are primarily interested in ultrafilters $U$ whose $Q_{U}$ 's enjoy this latter property.

Received by the editors December 22, 1986 and, in revised form, December 14, 1987.

1980 Mathematics Subject Classification (1985 Revision). Primary 03E05; Secondary 03E40, 03E35, 04A20. 
However, the slightly stronger property, that $Q_{U}$ is $d$-nondominating, gives slightly more information about $U$. Moreover the ultrafilters $U$ which we will construct having $Q_{U} b$-nondominating will in fact have a $d$-nondominating $Q_{U}$.

In the next section we will examine what other combinatorial properties $\lambda$ nondominating ultrafilters have. We remind the reader of several of these properties. An ultrafilter $U$ is called a $P$-point if each real in ${ }^{\omega} \omega$ is either finite-to-one or constant on a set in $U . U$ is a $Q$-point (respectively semi-Q-point) if for each $f: \omega \rightarrow \omega$, there is a set $\left\{x_{0}, x_{1}, \ldots\right\} \in U$ such that $\forall n \in \omega x_{n+1}>f\left(x_{n}\right)$ (respectively $\left.x_{n}>f(n)\right)$. Semi- $Q$-points have also been called rapid. All $Q$-points are clearly rapid, but not conversely. Ultrafilters which are both $P$-points and $Q$-points are called selective. The Continuum Hypothesis guarantees the existence of many selective ultrafilters, nonselective $P$-points, and nonselective $Q$-points. However, it is consistent with $c=\omega_{2}$ that there are no $Q$-points, and it is also consistent with $c=\omega_{2}$ that there are no $P$-points. If $f: \omega \rightarrow \omega$ is not constant on any set in an ultrafilter $U$, then $f_{*}(U)$ is defined to be $\left\{E \mid f^{-1}(E) \in U\right\}$. An ultrafilter $V$ precedes $U$ in the Rudin-Keisler ordering if $V=f_{*}(U)$. Selective ultrafilters are precisely those ultrafilters that have no predecessors in the Rudin-Keisler ordering. (The reader may consult $[\mathbf{4}, \mathbf{5}$ and 11] for a more detailed discussion of the various properties of ultrafilters, as well as for proofs of the facts cited above.)

Properties of nondominating ultrafilters. The following lemmas show that a $b$-nondominating ultrafilter is a $P$-point which has no rapid predecessors in the Rudin-Keisler order. The first two lemmas are included for the sake of completeness; their elementary proofs are left to the reader.

LEMMA 2. If $U \in V$ is rapid and $g: \omega \rightarrow \omega$ is an increasing function whose range is almost contained in each set from $U$, then $g$ eventually dominates all functions in $V$. Conversely, if $G$ is $Q_{U}$-generic over $V$ and its enumerating function eventually dominates all reals in $V$, then $U$ is rapid.

LEMMA 3. If $G$ is $Q_{U}$-generic over $V, f \in V$ is not constant on any set in $U$, and $W=f_{*}(U)$, then the set $f(G)$ is $Q_{W}$-generic over $V$. Hence each class of $\lambda$-nondominating ultrafilters is closed downward in the Rudin-Keisler order.

LEMMA 4. If an ultrafilter $U$ in $V$ is not a P-point, and $G$ is almost contained in every set in $U$, then in $V[G]$ there is a real $H: \omega \rightarrow \omega$ which eventually dominates all reals in $V$.

REMARK. In [3] Blass and Shelah credit P. Nyikos with independently discovering this result.

ProOF. In $V$, find a function $f: \omega \rightarrow \omega$ which is neither constant nor finiteto-one on any set in $U$. Let $A_{k}=\{a \mid f(a)>k\}$. Then $A_{k} \in U . G-A_{k}$ is finite, so we may define, in $V[G], h(k)=\max \{a \in G \mid f(a) \leq k\}$. Observe that $\forall a \in G$, $h[f(a)] \geq a$. Let $H(n)=h(\operatorname{Next}[f(G), n])$.

We show that $H$ dominates all reals $F$ in $V$. Let $F$ be any such real. We may assume that $F$ is increasing. Let $E=\{a \mid F(f(a))<a\} . f$ is finite-to-one on $\omega-E$; indeed $\left|f^{-1}(k) \cap(\omega-E)\right| \leq F(k)+1$. Hence $E \in U$. Let $m>\max (G-E)$. Let $m^{\prime}=\max \{f(0), f(1), \ldots, f(m)\}$. We will show that $H(n)>F(n)$ whenever $n>m^{\prime}$. 
Assume $n>m^{\prime}$. Let $k=\operatorname{Next}[f(G), n]$ and find $a \in G$ such that $f(a)=k$. $f(a)>m^{\prime}$ so $a>m$. Hence $a \in E$, and $F(k)=F[f(a)]<a$. However $n<k$ and $F$ is increasing so $F(n)<a$. Moreover $H(n)=h(k)=h[f(a)]>a$ since $a \in G$. Thus $H(n)>a>F(n)$.

If $U$ is an ultrafilter, then $U$ induces a linear-ordering $<_{U}$ on ${ }^{\omega} \omega$ where $f<_{U} g$ iff $\{n \mid f(n)<g(n)\} \in U$. We will write ${ }^{\omega} \omega / U$ for the structure consisting of $(U$ equivalence classes of) reals together with this ordering. The constant functions form an initial segment of ${ }^{\omega} \omega$; the remaining functions form the nonstandard part of ${ }^{\omega} \omega$. We consider the possible cofinality and coinitiality of this nonstandard part for $\lambda$-nondominating $U$ 's:

LEMMA 5. If $Q_{U}$ is $\lambda$-nondominating then the cofinality of ${ }^{\omega} \omega / U i s \geq \lambda$.

Proof. Let $C \subseteq{ }^{\omega} \omega / U$ be cofinal in ${ }^{\omega} \omega / U$. Working in $V[G]$, define for each $F \in C, H_{F}: \omega \rightarrow \omega$ by $H_{F}(n)=F[\operatorname{Next}(G, n)]$. We show that every real $f$ from $V$ is dominated by an $H_{F}, F \in C$ and hence, $|C| \geq \lambda$. Let $f$ be a real in $V$. We may assume $f$ is increasing. Find $F \in C$ such that $E=\{n \mid f(n)<F(n)\} \in U$ and find $m$ such that $\{n \in G \mid n>m\} \subseteq E$. Since $f$ is increasing, $f(n) \leq f[\operatorname{Next}(G, n)]$. But if $n>m, \operatorname{Next}(G, n) \in E$, hence $f[\operatorname{Next}(G, n)]<F[\operatorname{Next}(G, n)]=H_{F}(n)$. Thus $f(n)<H_{F}(n)$ whenever $n>m$.

LEMMA 6. If $Q_{U}$ is $\lambda$-nondominating then the nonstandard part of ${ }^{\omega} \omega / U$ had coinitiality $\geq \lambda$.

ProOF. Let $C \subseteq{ }^{\omega} \omega / U$ be coinitial in the nonstandard part of ${ }^{\omega} \omega / U$. For each $F \in C$ and each $k \in \omega,\{n \mid F(n)>k\} \in U$, hence almost includes $G$. In $V[G]$, define, $H_{F}: \omega \rightarrow \omega$ by $H_{F}(n)=\operatorname{Min}\{k \in G \mid \forall j \geq k j \in G$ implies $F(j)>n\}$. We show that each real $f$ from $V$ is dominated by an $H_{F}$. Again let $f$ be any increasing real in $V$. Let $h$ be the real which takes on value $n$ on the interval $[f(n-1), f(n))$ and value 0 on $[0, f(0))$. $h$ is not constant on any set in $U$, hence we may find $F \in C$ such that $E=\{n \mid F(n)<h(n)\} \in U$. Find $m \in G$ such that $\{a \in G \mid a \geq m\} \subseteq E$. For all but finitely many $n, f(n)>m$. We will show that for such $n, f(n)<H_{F}(n)$.

Assume $f(n)>m$. Let $B=\{a \in G \mid a \leq f(n)\}$. $B$ is finite, so let $k=\max (B)$. $m \in B$, so $k \geq m$. Thus $k \in E$ and hence $F(k)<h(k)$. Moreover $k \leq f(n)$ so $h(k) \leq n$. Thus $F(k)<n$ so $H_{F}(n)>k$. It is therefore impossible for $H_{F}(n) \leq$ $f(n)$, since this implies $H_{F}(n) \in B$ and $k=\max (B)$.

COROLlaRY 7. If $Q_{U}$ is d-nondominating, then the nonstandard part of ${ }^{\omega} \omega / U$ has cofinality and coinitiality $d$; hence $d$ must be regular.

PROOF. It is easy to show (see [4] for example) that this cofinality and coinitiality is always $\leq d$. By the lemma, this cofinality must actually $=d$ if $Q_{U}$ is $d$-nondominating.

We conclude this section by presenting a sufficient condition for $Q_{U}$ to be $d$ nondominating: that $U$ has a generating set of cardinality $<d$. In light of our previous results, this generalizes a theorem of Ketonen in [9] that a $U$ with a generating set of cardinality $<d$ must be a nonselective $P$-point, and a theorem of Blass in [1] that for such a $U$ the cofinality of ${ }^{\omega} \omega / U$ is $d$. The latter result follows from Corollary 7.

Our result is an immediate consequence of the following general lemma, which may be of some independent interest. 
LEMMA 8. If $Q$ is a partial order which has a dense set of cardinality $<d$, then $Q$ is d-nondominating.

COROLLARY 9. If $U$ is an ultrafilter generated by fewer than $d$ sets, then $Q_{U}$ is d-nondominating.

REMARKS. The consistency of the existence of such ultrafilters was established by Blass and Shelah in [2 and 3]. The converse of this corollary is clearly false: in the next section we will see that $d$-nondominating ultrafilters exist in models satisfying $\mathrm{CH}$. In such models a set of cardinality $<d$ is countable, and no such set can generate an ultrafilter.

PROOF OF LEMMA 8. We may assume that $|Q|<d$. Let $G$ be $Q$-generic over $V$ and let $D$ be a set of reals in $V[G]$ with $|D|<d$. Clearly $Q$ is $d$-c.c. so we may find, in $V$, a family $D^{\prime}$ of names for reals so that $D \subseteq D^{\prime}$ and $\left|D^{\prime}\right|<d$.

We now work in $V$. For each $q \in Q$ and each $\tau \in D^{\prime}$ let $g_{q, \tau}: \omega \rightarrow \omega$ by letting $g_{q, \tau}(n)$ be any element of $\{k: \exists p \leq q p \Vdash \tau(n)=k\}$. There are fewer than $d$ of these $g_{q, \tau}$ so we may find a real $g$ which is not dominated by any of them. We will show that

$$
\forall \tau \in D^{\prime} \forall m \in \omega \Vdash \exists n>m g(n) \geq \tau(n) .
$$

Let $\tau, m$ and $q \in Q$ be given. Find $n>m$ such that $g(n) \geq g_{q, \tau}(n)$. We may also find $p \leq q$ such that $p \Vdash \tau(n)=g_{q, \tau}(n)$. Then $p \Vdash g(n) \geq \tau(n)$.

The results of this section generalize two theorems about ultrafilters which are generated by fewer than $d$ sets: a theorem of Ketonen in [9] that a $U$ with a generating set of cardinality $<d$ must be nonselective $P$-point, and a theorem of Blass in [1] that for such a $U$ the cofinality of ${ }^{\omega} \omega / U$ is $d$. Since we have just shown that such ultrafilters are necessarily $d$-nondominating, the theorem of Blass follows immediately from Corollary 7. Moreover, since $d$-nondominating ultrafilters are clearly $b$-nondominating, they must be $P$-points which are not rapid, as shown in the beginning of this section. Since all selective ultrafilters are $Q$-points, and all $Q$-points are rapid, it is clear that $d$-nondominating ultrafilters cannot be selective and we obtain Ketonen's result: that ultrafilters generated by fewer than $d$-sets must be nonselective $P$-points.

\section{Existence theorem.}

THEOREM 10. Assume $d=c$. Then there exists an ultrafilter $U$ on $\omega$ with the property that $Q_{U}$-forcing adds no dominating real.

In particular, $b$-nondominating ultrafilters exist in models of the Continuum Hypothesis, or, more generally, Martin's Axiom. In [3], Blass and Shelah showed that such ultrafilters exist in a model obtained by adding Cohen reals. In these models $c=d$, so our theorem generalizes that result as well. This theorem shows that $d$-nondominating is stronger than $b$-nondominating, as there are models in which $c=d$ and $c$ is singular. The theorem guarantees that in such models $b$ nondominating ultrafilters exist, yet Corollary 7 precludes the existence of a $d$ nondominating ultrafilter in such a model.

Since Lemma 4 implies that $b$-nondominating ultrafilters must be $P$-points, Theorem 10 also generalizes another result of Ketonen, also in [9]: that $c=d$ implies the existence of a $P$-point. A Continuum Hypothesis construction of $P$-points with no $Q$-point predecessors in the Rudin-Keisler order was given by Daguenet in [6]. 
There the ultrafilter was constructed as the union of a $c$-length chain of filters, each of which was the union of a countable collection of compact subsets of $P(\omega)$. (Here, as in the rest of this paper, the topology on $P(\omega)$ is the standard, metric topology which arises by identifying $P(\omega)$ with ${ }^{\omega} 2$ and using the product topology on the latter.) We will prove our theorem in a similar way.

DEFINITION 11. A family of subsets of $\omega$ is said to be $K \alpha$ if it is a union of fewer than $\alpha$ families, each of which is compact. Note that for $\alpha>\omega$, a filter is $K \alpha$ if it has a $K \alpha$-generating set.

DEFINITION 12. A preterm is a partial map from $P \times \omega$ into $\omega$. If $U$ is an ultrafilter and $\tau$ is a term in the $Q_{U}$-forcing language, then the preterm $\hat{\tau}$ for $\tau$ is the partial map given by

$$
\hat{\tau}(s, n)=k \text { iff } \exists A \in U(s, A) \Vdash \tau(n)=k .
$$

$\hat{\tau}$ is a partial function since $U$ is closed under pairwise intersection. However $\hat{\tau}$ is not continuous in the sense that one may very well have, for some specific $\hat{\tau}, n \in \omega$, comparable $s, t \in P$ such that $(s, n) \in \operatorname{dom}(\hat{\tau}),(t, n) \in \operatorname{dom}(\hat{\tau})$ but $\hat{\tau}(s, n)$ and $\hat{\tau}(t, n)$ are not equal.

DEFINITION 13: A preterm $\hat{\tau}$ is said to be complete over a set $A$, if whenever $A$ is written as a finite union of sets $A=\bigcup\left\{A_{i} \mid i<k\right\}$, there is some $i<k$ satisfying

$$
\forall s \in P, \forall n \in \omega, \exists t<\left(s, A_{i}\right), \exists k, \hat{\tau}(t, n)=k .
$$

A preterm $\hat{\tau}$ is said to be complete over a family of sets iff it is complete over every set in the family.

LEMMA 15. If $U$ is an ultrafilter, and $\hat{\tau}$ is a preterm for a term $\tau$ in the $Q_{U^{-}}$ forcing language, then $\hat{\tau}$ is complete over $U$, hence also over every subfamily of $U$.

ProOF. Under the hypotheses, if $A \in U$ is a finite union of sets $A_{0}, A_{1}, \ldots, A_{k-1}$, then for some $i<k, A_{i} \in U$. We check that (14) holds for $A_{i}$. Given $s \in P$ and $n \in \omega$ we may find $(t, B) \leq\left(s, A_{i}\right)$ and $k \in \omega$ such that $(t, B) \Vdash \tau(n)=k$. Then $\hat{\tau}(t, n)=k$ and $t<\left(s, A_{i}\right)$.

LEMMA 16 (MAIN LEMMA). If $F$ is a $K \beta$ filter, for $\omega<\beta \leq d$ and $\hat{\tau}$ is a preterm which is complete over $F$, then there exists a $K \beta$ filter $F^{\prime}$ extending $F$ and $a h \in{ }^{\omega} \omega$ with the property that if $U$ is any ultrafilter extending $F^{\prime}$ and $\tau$ is any term in the $Q_{U}$-forcing language whose preterm is $\hat{\tau}$, then

$$
\Vdash_{U} \text { " } \tau \text { does not eventually dominate } h^{\prime \prime} .
$$

Proof of TheOREM 10 USING Lemma 16. Let $\left\langle\hat{\tau}_{\alpha} \mid \alpha \in d\right\rangle$ enumerate the collection of all preterms. We will construct an increasing sequence of $K d$ filters $\left\langle F_{\alpha} \mid \alpha \in d\right\rangle$ and a sequence of functions $\left\langle h_{\alpha}: \alpha \in d\right\rangle$ which satisfy the following conditions:

1. $F_{\alpha}$ is $K\left[(\omega+|\alpha|)^{+}\right]$.

2. If $\beta<\alpha$ then $F_{\beta} \subseteq F_{\alpha}$.

3. If $U$ is any ultrafilter extending $F_{\alpha+1}$ and $\tau$ is any term in the $Q_{U}$-forcing language with preterm $\hat{\tau}_{\alpha}$, then

$$
\Vdash_{U} \text { " } \tau \text { does not eventually dominate } h_{\alpha} \text { ". }
$$


$F_{0}$ is the filter of cofinite sets. For limit ordinals $\alpha$, we let $F_{\alpha}=\bigcup\left\{F_{\beta} \mid \beta<\alpha\right\}$. At successor stages, we apply 16 to construct $g_{\alpha}$ and $F_{\alpha+1}$ satisfying the conditions. Condition 1 is used as an induction hypothesis.

We may then take for $U$ any ultrafilter which contains the union of the $F_{\alpha}$ 's. To check that $U$ has the desired properties, it is sufficient to observe that any term $\tau$ in the $Q_{U}$-forcing language will have its preterm $\hat{\tau}$ appear as one of the $\hat{\tau}_{\alpha}$ and will necessarily be complete over $F_{\alpha}$.

The remainder of this section will be devoted to proving the Main Lemma (Lemma 16). For the rest of the section we assume that $\hat{\tau}$ is some fixed preterm. Fix an enumeration of $P$ so that $P=\left\{s_{i}: i \in \omega\right\}$. For each $j, n, k \in \omega$ and $s \in P$, we define

$$
\begin{aligned}
\operatorname{Thick}(s, n, k) & =\{A \subseteq \omega \mid \exists t<(s, A) \exists i<k \hat{\tau}(t, n)=i\} \\
\operatorname{Thin}(s, n, k) & =P(\omega)-\operatorname{Thick}(s, n, k) \\
\operatorname{Bad}(j, k) & =\left\{\bigcup_{[m<j]} B_{m}: \forall m<j \exists i<j B_{m} \in \operatorname{Thin}\left(s_{i}, j, k\right)\right\} .
\end{aligned}
$$

We will refer to the members of $\operatorname{Thick}(s, n, k)$, $\operatorname{Thin}(s, n, k)$ and $\operatorname{Bad}(j, k)$ as $(s, n, k)$-Thick, $(s, n, k)$-Thin, and $(j, k)$-Bad sets, respectively, and add the language "with respect to (w.r.t) $\hat{\tau}$ " when more than one preterm is under discussion. (This will not be necessary in this section.) A set is $(j, k)$-Bad if it the union of at most $j$ sets which are each, for some $i<j,\left(s_{i}, j, k\right)$-Thin. We observe that Thick $(s, n, k)$ is always open and hence $\operatorname{Thin}(s, n, k)$ and $\operatorname{Bad}(j, k)$ are always closed. We note also that Thick $(s, n, k)$ is closed under superset, and hence Thin $(s, n, k)$ is closed under subset.

LEMMA 17. For each $j \in \omega$ and $A \subseteq \omega$ such that $\hat{\tau}$ is complete over $A$, there exists $k \in \omega$ such that $A$ is not $(j, k)-B a d$.

ProOF. It will be sufficient if we pick $k$ so that $A$ cannot be partitioned into $j$ sets, each of which is $\left(s_{i}, j, k\right)$-Thin for some $i<j$.

Let $A=\left\{a_{i} \mid i \in \omega\right\}$. Let $T$ be the tree $T=\left\{{ }^{n} j \mid n \in \omega\right\}$. We identify the nodes of $T$ with partitions of initial segments of $A$ into $j$ pieces as follows: If $p \in{ }^{n} j$, let $M(p, i)=\left\{a_{m} \mid m<n \& p(m)=i\right\}$, and let $H_{p}=\{M(p, i) \mid i<n\}$. The $H_{p}$ 's give us all partitions of initial segments of $A$ into $j$ sets and give us a correspondence between paths through the tree and partitions of $A$ itself.

We will now assign to each node $p$ of $T$ a natural number $k(p)$ by applying the rules given below. Intuitively, the $k(p)$ 's represent approximations to $k . k(p)$ is chosen in such a way that if a partition of $A$ "extends" $H_{p}$, at least one of the sets in the partition will be $\left(s_{i}, j, k(p)\right)$-Thick for all $i<j$. The rules for calculating $k(p)$ are given below in the order in which they should be applied:

1. If there is a set $M \in H_{p}$ which has the property that for every $i<j$ there exist $r_{i}<\left(s_{i}, M\right)$ and $m_{i}$ for which $\hat{\tau}\left(r_{i}, j\right)=m_{i}$, then we select one such $M$ and set $k(p)$ to be the maximum of its associated $m_{i}$ 's.

2. Otherwise, if each immediate successor $p^{\prime}$ of $p$ has been assigned a value $k\left(p^{\prime}\right)$, then set $k(p)$ equal to the maximum of all these $k\left(p^{\prime}\right)$.

Call a node $p$ definite if $k(p)$ is defined. It should be clear that if a node is definite then all its successors are also definite; indeed, the same $k$ may be used for 
all the successors. Unfortunately, it is less obvious that the root node (and hence every node) is definite. Indeed this is where completeness is used.

Assume, for contradiction, that the root node is not definite. At least one of its immediate successors is not definite. Pick one such node and observe that at least one of its successors is not definite. By continuing in this way, we obtain a path $F: \omega \rightarrow j$ through $T$ with the property that none of the nodes on this path are definite. For all $i<j$, let $A_{i}=\left\{a_{n} \mid F(n)=i\right\}$. Then $A=\bigcup\left\{A_{i} \mid i<j\right\}$. By the completeness of $\hat{\tau}$, we may find $i<j$ such that (14) holds. Hence for each $n<j$, we may find $m_{n}$ and $r_{n}<\left(s_{n}, A\right)$. such that $\hat{\tau}\left(r_{n}, j\right)=m_{n}$. Let $m^{\prime}=\max \left\{m_{n} \mid n<j\right\}$. Let $M=\bigcup\left\{\right.$ Range $\left.\left[r_{n}-s_{n}\right] \mid n<j\right\} . M$ is a finite subset of $A_{i}$; hence there is a node $p$ on the path $F$ such that $M \in[H(p)]$. We also have $r_{n}<\left(s_{n}, M\right)$ for all $n<j$. Hence $p$ is definite; we may take $k(p)$ to be $m^{\prime}$. This contradicts the fact that no node on $F$ is definite.

To complete the proof, take $k$ to be the value assigned to the root node.

LEMMA 18. Given $j \in \omega$ and $K$ a compact family of sets such that $\hat{\tau}$ is complete over $K$, there exists $k \in \omega$ such that no $A$ in $K$ is $(j, k)-B a d$.

Proof. For each $k \in \omega$, let $O_{k}=\{A \subseteq \omega \mid A$ is not $(j, k)$-Bad $\}$. By Lemma 17, the family $\left\{O_{k}: k \in \omega\right\}$ covers $K$. But the $O_{k}$ 's are open and increasing; hence a single $O_{k}$ will cover $K$.

ProOF OF MAIN LEMMA (16). Let $F=\bigcup\left\{K_{\alpha} \mid \alpha<\lambda\right\}$, where $\lambda<\beta$ and each $K_{\alpha}$ is a compact family. We must construct a $K \beta$ filter $F^{\prime}$ extending $F$ and a $h \in{ }^{\omega} \omega$ such that if $U$ is any ultrafilter extending $F^{\prime}$ and $\tau$ is any term in the $Q_{U}$-forcing language whose preterm is $\hat{\tau}$, then

\section{$\Vdash_{U}$ " $\tau$ does not eventually dominate $h$ ".}

Use the previous lemma to construct, for each $\alpha<\beta$, a function $h_{\alpha}: \omega \rightarrow \omega$, such that

$$
\forall A \in K_{\alpha} \forall j \in \omega A \text { is not }\left(j, h_{\alpha}[j]\right) \text {-Bad. }
$$

Find a single $h: \omega \rightarrow \omega$ which is not dominated by any of the $h_{\alpha}$ 's. Let

$$
I[h]=\{B \subseteq \omega \mid \exists s \in P, \exists m \in \omega, \forall n>m, B \text { is }(s, n, h(n)) \text {-Thin }\} .
$$

$I[h]$ is a countable union of compact families which is closed under subset. We will let $F^{\prime}$ be the filter generated by $F \cup\{\omega-B \mid B \in I[h]\}$. First we must verify that this family has the Finite Intersection Property.

Assume, by way of contradiction, that we are given a finite family of sets from $F \cup\{\omega-B \mid B \in I[h]\}$ which has void intersection. We may then obtain a single set $A \in F$ and a finite subfamily, $\left\{B_{k}, k<n\right\}$ of $I[h]$, such that $A=\bigcup\left\{B_{k}, k<n\right\}$. Associated with each $B_{k}$ there is a condition $s_{i(k)}$ from $P$ and an $m_{k} \in \omega$ such that $B_{k}$ is $\left(s_{i}, m, h[m]\right)$-Thin for all $m>m_{k}$. Finally, find $\alpha$ such that $A \in K_{\alpha}$. Pick $j$ so large that
1. $h(j)>h_{\alpha}(j)$.
2. $j>n$.
3. $\forall k<n, j>m_{k}$.
4. $\forall k<n, j>i(k)$. 
Such a $j$ exists since the first condition holds for infinitely many $j$ while the others hold for all but finitely many $j$. However, for such a $j$, each $B_{k}$ is $\left(s_{i}, j, h_{\alpha}[j]\right)$ Thin. There are fewer than $j$ of them, hence their union, $A$, is $\left(j, h_{\alpha}[j]\right)$-Bad. This contradicts (19) and hence establishes the FIP.

Finally, to verify that $F^{\prime}$ has the desired property, we let $U$ be any ultrafilter extending $F$ and $\tau$ be a term in $Q_{U}$-forcing language and we check that

$$
\Vdash_{U} \text { " } \tau \text { does not eventually dominate } h \text { ". }
$$

For contradiction, assume that we have $(s, E) \in Q_{U}$ and $m \in \omega$ such that

$$
\forall n>m(s, E) \Vdash \tau(n)>h(n) .
$$

Since $E \in U$, we have that $E$ is not in $I(h)$. Hence we may find $n>m$ such that $E$ is not $(s, n, h(n))$-Thin. Thus there is a $t<(s, E)$ and $i<h(n)$ such that $\hat{\tau}(t, n)=i$. Then for some $B \in U$ such that $(t, B) \Vdash \tau(n)=i$. However $(t, B \cap E) \Vdash \tau(n)=h(n)$. This gives us the contradiction needed to complete the proof of the Main Lemma [Lemma 16].

\section{The stronger property.}

THEOREM 20. If $\left|\left\{X c^{\omega} \omega:|X|<d\right\}\right|=d$, then there exists an ultrafilter $U$ on $\omega$ with the property that $Q_{U}$-forcing is d-nondominating.

The hypothesis implies that $c=d$ and that $d$ is regular. The latter is a necessary condition in light of Corollary 7. Before proving the theorem we need to introduce some additional terminology. The maximum of a finite set of preterms $\left\{\hat{\tau}_{0}, \hat{\tau}_{1}, \ldots, \hat{\tau}_{n}\right\}$ is defined to be the preterm $\hat{\tau}^{\prime}$ whose domain is the intersection of the domains of $\hat{\tau}_{0}, \hat{\tau}_{1}, \ldots, \hat{\tau}_{n}$ and whose value at any point $(s, k)$ of its domain is the maximum of $\left\{\hat{\tau}_{0}(s, k), \hat{\tau}_{1}(s, k), \ldots, \hat{\tau}_{n}(s, k)\right\}$. Note that a set which is $(s, n, k)$-Thick with respect to $\hat{\tau}^{\prime}$ is necessarily $(s, n, k)$-Thick w.r.t. each $\hat{\tau}_{i}$. Hence a set which is $(s, n, k)$-Thin w.r.t any $\hat{\tau}_{i}$ is also $(s, n, k)$-Thin w.r.t. $\hat{\tau}^{\prime}$. When $\hat{\tau}^{\prime}$ is complete over a filter, we will say that the set $\left\{\hat{\tau}_{o}, \hat{\tau}_{1}, \ldots, \hat{\tau}_{n}\right\}$ is simultaneously complete over the filter.

LEMMA 21. If $F$ is a Kd filter and $T$ is any collection of preterms of cardinality $<d$ which has the property that any finite subset of $T$ is simultaneously complete over $F$, then there exists a $K \beta$ filter $F^{\prime}$ extending $F$ and $a h \in{ }^{\omega} \omega$ with the property that if $U$ is any ultrafilter extending $F^{\prime}$ and $\tau$ is any term in the $Q_{U}$-forcing language with a preterm $\hat{\tau}$ in $T$, then

$$
\Vdash_{U} \text { " } \tau \text { does not eventually dominate } h^{\prime} \text {. }
$$

SKetch OF PROOF. The proof is quite similar to the proof of Lemma 16; we briefly describe the necessary modifications. We may assume that $T$ is closed under finite maxima, and that each preterm in $T$ is complete over $F$. We again let $F=\bigcup\left\{K_{\alpha} \mid \alpha<\beta\right\}$, where each $K_{\alpha}$ is a compact family. We construct, for each $\alpha<\beta$ and each $\hat{\tau} \in T$ a function $h_{\alpha, \tau}: \omega \rightarrow \omega$, such that

$$
\forall A \in K_{\alpha} \forall j \in \omega A \text { is not }\left(j, h_{\alpha, \tau}[j]\right) \text {-Bad w.r.t. } \hat{\tau} \text {. }
$$

Find a single $h: \omega \rightarrow \omega$ which is not dominated by any of the $h_{\alpha, \tau}$ 's. Let

$$
I[h]=\{B \subseteq \omega \mid \exists \hat{\tau} \in T, \exists s \in P, \exists m \in \omega, \forall n>m, B \text { is }(s, n, h[n]) \text {-Thin w.r.t. } \hat{\tau}\} .
$$


$I[h]$ is a $K d$ family, and so is $F \subset\{\omega-B \mid B \in I[h]\}$. The crucial step is again the verification that this family has the FIP. If it does not, we may find a single set $A \in F$, a finite subfamily $\left\{B_{k}, k<n\right\}$ of $I[h]$, and associated $\hat{\tau}_{k}, s_{i(k)}$, an $m_{k}$ such that $A=\bigcup\left\{B_{k}, k<n\right\}$ and such that each $B_{k}$ is, $\forall m>m_{k},\left(s_{i}, m, h[m]\right)$-Thin with respect to $\hat{\tau}_{k}$. Let $\hat{\tau}^{\prime}$ be the maximum of $\left\{\hat{\tau}_{0}, \hat{\tau}_{1}, \ldots, \hat{\tau}_{n}\right\}$ so that each $B_{k}$ is also $\left(s_{i}, m, h[m]\right)$-Thin w.r.t. $\hat{\tau}^{\prime}$. Find $\alpha$ such that $A \in K_{\alpha}$. Again there are infinitely many $j$ for which $h(j)>h_{\alpha, \tau^{\prime}}(j)$, so we may find one $j>n$ such that each $B_{k}$ is $\left(s_{i}, j, h_{\alpha, \tau^{\prime}}(j)\right)$-Thin with respect to $\hat{\tau}^{\prime}$. Their union $A$ will then be $\left(j, h_{\alpha, \tau^{\prime}}(j)\right)$-Bad with respect to $\hat{\tau}^{\prime}$. This contradicts the choice of $h_{\alpha, \tau^{\prime}}$ and establishes the lemma.

LEMMA 22. If $U$ is an ultrafilter, $\left\{\tau_{0}, \tau_{1}, \ldots, \tau_{n}\right\}$ are terms in the $Q_{U}$-forcing language and $\hat{\tau}_{0}, \hat{\tau}_{1}, \ldots, \hat{\tau}_{n}$ are their respective preterms, then $\left\{\hat{\tau}_{0}, \hat{\tau}_{1}, \ldots, \hat{\tau}_{n}\right\}$ is simultaneously complete over $U$ and hence over every subfamily of $U$.

ProOF. We proceed as in the proof of Lemma 15. If $A \in U$ is a finite union of sets $A_{0}, A_{1}, \ldots, A_{k-1}$, then for some $i<k, A_{i} \in U$. Given $s \in P$ and $m \in \omega$, we seek $t<\left(s, A_{i}\right)$ such that $(t, m) \in \bigcap\left\{\operatorname{dom}\left(\hat{\tau}_{i}: i<n\right)\right\}$. We may, however, find $(t, B) \leq\left(s, A_{i}\right)$ and $k_{0}, k_{1}, \ldots, k_{n} \in \omega$ such that

$$
(t, B) \Vdash\left[\tau_{0}(m)=k_{0} \& \tau_{1}(m)=k_{1} \cdots \& \tau_{n}(m)=k_{n}\right]
$$

which implies that $(t, m) \in \bigcap\left\{\operatorname{dom}\left(\hat{\tau}_{i}\right): i<n\right\}$.

SKETCH OF PROOF OF THEOREM 20. This proof is similar to the proof of Theorem 16; again we briefly describe the differences. $U$ will again be the union of a sequence of $K d$ filters $\left\langle F_{\alpha} \mid \alpha \in d\right\rangle$. Let $\left\langle T_{\alpha} \mid \alpha \in d\right\rangle$ enumerate the set of all collections of preterms of cardinality $<d$. At stage $\alpha$, if every finite subfamily of $T_{\alpha}$ is simultaneously complete over $F_{\alpha}$, we apply Lemma 21 and obtain a $K d$ filter $F_{\alpha+1}$ extending $F_{\alpha}$ and a real $h_{\alpha}$ such that

If $U$ is any ultrafilter extending $F_{\alpha+1}$ and $\tau$ is any term in the $Q_{U}$-forcing language with preterm $\hat{\tau} \in T_{\alpha}$, then

$$
\Vdash_{U} \text { " } \tau \text { does not eventually dominate } h_{\alpha} \text { ". }
$$

We now verify that the $U$ thus produced is $d$-nondominating. If $D$ is any family of reals in $V[G]$ of cardinality $<d$ we may find a set $T$ of preterms in $V$ with $|T|<d$ such that each real in $D$ has a term whose preterm is in $T$. $T$ will appear as one of our $T_{\alpha}$, and each finite subfamily of it will be simultaneously complete over $F_{\alpha}$. Then (23) implies that $h_{\alpha}$ is not dominated by any real in $D$.

\section{Open questions.}

1. Is it our hypothesis that $c=d$ necessary? Is it consistent to have $c<d$ and still have the existence of $d$-nondominating, or even $b$-nondominating ultrafilters?

2. Are there $P$-point ultrafilters which have no rapid predecessors and are not $b$-nondominating?

3. Does the existence of a $d$-nondominating ultrafilter follow from the weaker hypothesis that $c=d$ and $c$ is regular? 


\section{REFERENCES}

1. A. Blass, Near coherence of filters, I. Cofinal equivalence of models of arithmetic, Notre Dame J. Formal Logic 27 (1986), 579-591.

2. A. Blass and S. Shelah, Near coherence of filters. III. A simplified consistency proof, Notre Dame J. Formal Logic (to appear).

3. __ Ultrafilters with small generating sets, 73 (1988), 1-79.

4. R. M. Canjar, Countable ultraproducts without CH, Ann. Pure and Appl. Logic (to appear).

5. W. W. Comfort and S. Negrepontis, Theory of ultrafilters, Springer-Verlag, Berlin, Heidelberg and New York, 1974.

6. M. Daguenet, Propriete de Baire de $\beta N$ muni d'une nouvelle topologie et application a la construction des ultrafiltres, Sem. Choquet, $14^{e}$ annee, 1974/75.

7. S. Hechler, $A$ dozen small uncountable cardinals, TOPO 72-General Topology and its Applications, Lecture Notes in Math., vol. 378, Springer-Verlag, Berlin, Heidelberg and New York, 1972, pp. 207-218.

8. _ A ubiquitous cardinal, Proc. Amer. Math. Soc. 52 (1975), 348-352.

9. J. Ketonen, On the existence of P-points, Fund. Math., 92 (1976), 91-99.

10. A. Mathias, Happy families, Ann. Math. Logic 12 (1977), 59-111.

11. A. Miller, There are no Q-points in Laver's model for the Borel conjecture, Proc. Amer. Math. Soc. 78 (1980), 103-106.

12. C. Puritz, Skies, constellations, and monads, Contributions to Non-Standard Analysis (W. A. J. Luxemburg and A. Robinson, eds.), North-Holland, 1972

13. E. van Douwen, The integers and topology, Handbook of Set-Theoretic Topology, NorthHolland, 1984.

Department of Computer SCIEnCE, Mathematics and Statistics, University OF BALTIMORE, BALTIMORE, MARYLAND 21201 